\title{
Learning as the creation of stable representations of stimuli
}

\author{
Luca Dellanna
}

\begin{abstract}
First, this paper explains how learning depends on the ability of producing stable representations of stimuli. Then, it shows how most learning disabilities derive from an inability to produce such stable representations of stimuli. Finally, it demonstrates how learning can be quantified as the reduction of entropy in the representation of stimuli.
\end{abstract}

\section{How learning works}

The are four fundamental concepts about learning:

1. Learning is about detecting patterns. We learn to read by recognizing that patterns of neural activation in our retina correspond to lines on a sheet of paper, that patterns of lines form letters and that patterns of letters form words.

2. Learning is about creating stable representations of stimuli patterns. For example, in order to learn to read handwriting, we need to understand that all shapes which are similar to a spike or a vertical line with a dot on top represent the letter " $\mathrm{i}$ " - even if some are slightly longer or slightly taller. The ability to create a stable representation ("the letter i") out of variations of an unstable stimulus (the exact shape of how the "i" has been written) is a prerequisite for learning.

3. Moreover, learning is about creating stable representations of stimuli patterns in hierarchical order. We need to learn the patterns of lines that form letters before we can learn the patterns of letters that form words. I will call "lower-order representations" the patterns which have to learn first, in order to later enable the learning of "higher-order representations (following the previous example, letters are a lower-order representation of the lines that form writing; words are a high-order one).

4. Finally, noise prevents learning. It is much easier to learn how to read if exposed to printed text rather than to handwriting only (the latter being much more noisy - each given letter is drawn slightly differently each time).

Email: Luca@luca-dellanna.com

Website: www.luca-dellanna.com

Twitter: @dellannaluca
Putting these four concepts together, it follows that learning the lower-order representations of stimuli is necessary in order to start learning higher-order ones; moreover, the learning of higher-order representations of stimuli cannot start before the representation of lower-order ones is stable enough (i.e., non-noisy). Only once lower-order representations of stimuli are learned properly, learning of higher-order ones can start. For example, an individual cannot learn how to read and spell words unless he has learnt before how to perceive letters in a stable, non-noisy way. If you are not convinced, imagine how it would be to learn to read and write in a new language if, any time you read anything in the new language, one out of five characters was random (a form of noise introduced to simulate the fact that you didn't learn yet how to properly distinguish letters). Noise at the letter level would prevent you from learning patterns at the word level.

If a lower region of our brain is unable (for any reason) to create a stable representation of the stimuli it receives, then its output will be noisy and unstable. In this case, an higher region of our brain will not be able to detect any pattern in the output of the noisy lower region and will therefore be unable to learn anything from it.

\section{Learning obstacles}

There are three classes of obstacles that prevent creation of stable representations of stimuli and concepts (Table 1).

\begin{tabular}{|l|l|l|l|}
\hline Underlying cause & Resulting obstacle & $\begin{array}{l}\text { Real world } \\
\text { example }\end{array}$ & Potential solution \\
\hline $\begin{array}{l}\text { Malfunction in sensorial } \\
\text { apparatus }\end{array}$ & $\begin{array}{l}\text { Stimuli generation is } \\
\text { noisy }\end{array}$ & $\begin{array}{l}\text { Individuals with } \\
\text { myopia }\end{array}$ & $\begin{array}{l}\text { Spectacles - filters out } \\
\text { noise so clean visual stimuli } \\
\text { is received }\end{array}$ \\
\hline Not enough practice & $\begin{array}{l}\text { Cannot distinguish } \\
\text { noise from signal }\end{array}$ & $\begin{array}{l}\text { Learning new } \\
\text { languages }\end{array}$ & $\begin{array}{l}\text { Increased practice \& } \\
\text { exposure to understand } \\
\text { what is noise and what is } \\
\text { signal }\end{array}$ \\
\hline $\begin{array}{l}\text { Inability to appropriately } \\
\text { consider contextual } \\
\text { stimuli }\end{array}$ & $\begin{array}{l}\text { Does not receive some } \\
\text { critical stimuli or } \\
\text { receives too many } \\
\text { stimuli which carry no } \\
\text { signal }\end{array}$ & $\begin{array}{l}\text { Individuals with } \\
\text { Autism Spectrum } \\
\text { Disorder }\end{array}$ & $\begin{array}{l}\text { Progressive training to } \\
\text { decouple signal from noise } \\
\text { and allow the creation of } \\
\text { stable lower-order } \\
\text { representations of } \\
\text { fundamental concepts }\end{array}$ \\
\hline
\end{tabular}

Figure 1. The three classes of obstacles that impair learning 
The first cause usually leads to permanent learning disability unless compensated through technology (for example, spectacles allow the brains of patients with myopia to receive non-noisy visual stimuli).

The second cause is usually overcame over time, with repeated exposition to stimuli followed by learning. For example, this is how a child learns to speak by simple exposure to spoken language.

The third cause is usually the cause of a group of learning disabilities usually classified under the label of "Autism Spectrum Disorder" or ASD.

\section{Autism Spectrum Disorder (ASD): an impairment in cre- ating stable representations in fields where contextual stimuli matters}

The brains of people affected by ASD tend to disregard contextual stimuli. This is advantageous in fields where context carries no information, such as the learning of physics and computer science: it allows one to focus on individual items whose validity is independent of their context (which is noise). However, disregarding contextual data is disadvantageous in fields where context does carry information namely, learning social skills. For example, reading a transcript of a face-to-face interaction between two people is inadequate to fully understand what has been communicated since other cues such as tone of voice and non-verbal communication e.g. body language could have dramatically affected the meaning of any message being conveyed. The brain of a person affected by ASD tends to disregard contextual data such as intonation or body language and is therefore unable to receive all the relevant inputs needed to create stable representations of social interactions; therefore, his representations of social interactions will be noisy and unstable, thereby making it challenging for higher-order concepts to be formed - such as individual theories of human behavior (such as social norms and expectations regarding social interactions).

Similarly, people affected by ASD are generally less proficient than non-affected individuals at sports and reading facial expressions because muscle movements are largely contextual. Our muscles work in concert. In order to perform correctly an action such as jump or a smile, the individual movement of a single muscle is not so important; what matters is that multiple muscles work together in a coordinated movement. An individual with ASD tends to disregard context and to only focus on the movement of single muscles at a time. By missing the signal (the relative movements of the relevant muscles) and by only focusing on noise (the absolute contraction of a single muscle), an individual with ASD tends to create noisy and unstable representations of movements. As a consequence, he is impaired in learning anything based on such representations - such as more complex movements, or on learning their nuances.

My previous paper (Dell'Anna, 2018) explains in further detail how ASD is linked to an impairment in stable representations of concepts heavily dependant on context. In the same paper, I also hypothesized the existence of another learning disorder, which is fundamentally the opposite of ASD. People affected by it would have a neurological predisposition to overly relay on contextual data to make sense of the world leading to an impairment of mastering fields where context is noise, such as physics and computer science. The reason why such learning disorder would have gone undetected so far is that social interactions are more necessary than the knowledge of physics; therefore, an impairment in the former is easier to detect whereas an impairment in the latter might be misclassified as disinterest.

\section{Learning as the reduction of entropy in the representation of stimuli}

Finally, a parallel can be drawn between learning and the reduction of entropy. Entropy can be defined as "the quantity of information that is necessary to fully describe a given entity". Stable entities tend to have low entropy, whereas unstable entities tend to have high one - more information is needed to describe the variations between their unstable states.

The first part of this paper explained how learning causes incoming stimuli to be represented in a stabler way. It follows that learning causes a decrease in the entropy of the representation of stimuli. This might be important for fields that need a quantitative measure of learning for their models (such as machine learning).

\section{Conclusions}

First, I explained how learning depends on the ability of producing stable representations of stimuli. Then, I showed that most learning disabilities derive from an inability to produce such stable representations of stimuli. Finally, I demonstrated how learning can be quantified as the reduction of entropy in the representation of stimuli.

\section{References}

Dell'Anna, L. (2018, Feb). The high-pass filter hypothesis for autism spectrum disorder (asd). Retrieved from psyarxiv.com/xm5ca doi: 10.31234/osf.io/xm5ca 